\title{
DRUG PRESCRIBING PATTERN FOR UNDER-FIVES IN A PAEDIATRIC CLINIC IN SOUTH-WESTERN NIGERIA
}

\author{
Fadare Joseph $^{1}$, Olatunya Oladele ${ }^{2}$, Oluwayemi Oludare ${ }^{2}$, Ogundare Olatunde $^{2}$
}

\section{ABSTRACT}

BACKGROUND: The audit of drug prescribing pattern in under-five children is essential in identifying the various types of non-rational prescribing such as polypharmacy and irrational use of antimicrobials. The primary objective of this study was to determine the drug prescribing pattern for children aged below five years attending the paediatric outpatient clinic of a tertiary hospital in Ado-Ekiti, South-West Nigeria.

METHODS: A cross-sectional study was carried out using the medical records of patients who attended the paediatric clinic of the hospital between April 1 and October 30, 2013. The medical records of patients aged below five years were selected monthly for the period of the study using a regular interval ratio. Drug use indicators were assessed using the WHO guidelines on investigation of drug use in health care facilities.

RESULTS: There were 293(55.7\%) prescriptions collected from male patients and $233(44.3 \%$ from female patients with a total of 1369 prescribed drugs $(2.6 \pm 1.1$ drugs per prescription). A total of three hundred and seventy-four (71.1\%) patients had at least one antibiotic prescribed with antibiotics accounting for $28.2 \%$ of all drugs prescribed, while $13.5 \%$ of all encounters had an injection prescribed. Prescribing by generic name was done in $68.9 \pm 26.5 \%$ of all prescribed medications, and $60.4 \%$ of all prescribed medications were from the latest version of the Nigerian Essential Drug List. Three hundred and twelve children (59.3\%) had at least one anti-malarial drug prescribed while analgesics drugs accounted for $6.1 \%$ of all prescriptions.

CONCLUSION: This study showed that over-prescription of antibiotics, significant use of injections and prescribing by generic name are real issues among Nigerian paediatric prescribers.

KEYWORDS: Prescribing indicators, under-five, outpatients' clinic, rational prescribing, Nigeria

DOI: http://dx.doi.org/10.4314/ejhs.v25i1.10

\section{INTRODUCTION}

Children are a large proportions of the population of many developing countries, hence the importance of their health status. In Nigeria, according to the 2006 national census, children aged below 14 years constituted more than $40 \%$ of the population (1). Children aged five years and below constitute the bulk of patients attending the paediatric outpatient clinics in Nigeria (2). They are particularly vulnerable to many communicable /infectious diseases, and mortality among this group is usually very high, hence the need for special attention towards their care. When prescribing for children, there is a need to consider the aetiopathogenesis of the disease conditions as well as the developmental stage of the child at that particular time. Prescriptions can be described as rational and non-rational with the latter having possible consequences such as adverse drug reactions, increased frequency of drug-drug interactions and increased healthcare costs (3).

Adverse drug reactions in children particularly could contribute to morbidity and mortality because of the immaturity of their immune system and their inability to communicate adequately.

\footnotetext{
${ }^{1}$ Department of Pharmacology, Ekiti State University, Ado-Ekiti, Nigeria

${ }^{2}$ Department of Paediatrics, Ekiti State University, Ado-Ekiti, Nigeria.

Corresponding Author: Joseph O. Fadare, Email: jofadare@gmail.com
} 
Regular audit of prescriptions is essential in identifying the various types of non-rational prescribing such as polypharmacy, irrational use of antimicrobials and injectables among others (4). It is also necessary to describe trends and follow adherence to various treatment guidelines. Irrational use of antimicrobials has been identified as a major problem in many pediatric prescription studies; this could lead to antimicrobial resistance, treatment failures and increased healthcare costs (5).

Polypharmacy and other forms of inappropriate forms of prescribing could be extremely harmful in children because of their physiological peculiarities. The World Health Organization (WHO) has tried to address these non-rational practices through its resource "How to Investigate Drug Use in Health Facilities: Selected Drug Use Indicators", which was published in 1993 (6). The drug use indicators listed in this manual include prescribing, patient care and health facility indicators. The average number of prescribed drugs, percentage of prescribed drugs by generic name and percentage of encounters with antibiotics are some of the prescribing indicators. Other indicators in this group are percentage of drugs prescribed from essential drug list and percentage of encounter with injection.

In Nigeria, many prescription audits have been conducted mainly among adult patients attending the medical and general outpatient clinics of secondary and tertiary healthcare centers with various outcomes. However, the few available studies on pediatric prescribing pattern are old, and as a result, there is a need to assess the current practice, hence the rationale for this study.

The primary objective of this study was to determine the prescribing pattern of children aged below five years who were attending the pediatric outpatient clinic of a tertiary hospital in Ado-Ekiti, South-West Nigeria. Secondary objectives included the morbidity pattern and detailed analysis of prescribed antimicrobial and antimalarial medications; to assess whether rational or following the national malaria treatment policy

\section{METHODS}

Study Setting: A cross-sectional retrospective study was carried out using the medical records of patients who attended the pediatric outpatients' clinic of the teaching hospital between April 1 and October 30, 2013. The study was carried out in the pediatric outpatient clinic of the Ekiti State University Teaching Hospital, Ado-Ekiti, SouthWest Nigeria. The hospital is one of the two tertiary healthcare facilities in Ekiti State that provide medical care for its populace and that of the neighboring states. The hospital is well staffed with consultants in various fields of Pediatrics, Internal Medicine, Surgery, Obstetrics and Gynecology, Psychiatry, Community Medicine, Oral and Maxillofacial Surgery and Restorative Dentistry. The Pediatric Department is well staffed with its components of nursing staff, house officers, medical officers, registrars and consultants. Its outpatient clinic is usually the first point of contact for pediatric patients brought directly from home or referred from other healthcare facilities.

Sampling procedure: Using the WHO model of drug utilization study, a total of 600 patient records were selected for the six month period by systematic sampling. The attendance register of the pediatric outpatient clinic was retrieved and the medical records of 100 patients aged below five years were selected monthly for the period of the study using a regular interval ratio. The information retrieved from the case notes included bio-demographic data, working diagnoses, list of prescribed drugs and their routes of administration. The following drug use indicators were assessed using the WHO guidelines on investigation of drug use in health care facilities: average number of drugs per prescription, percentage of encounters with antibiotics, percentage of drugs prescribed by generic name, percentage of drugs prescribed from essential drug list and percentage of encounters with injection(6). The percentage of encounters with anti-malarial medications was also included because the study was conducted in a holoendemic malarial environment where malaria is responsible for significant morbidity and mortality among children especially those aged below five years. 
Statistical Analysis: Data collected was analyzed using Statistical Package for Social Sciences version 17 software (IBM Corporation, Armonk, NY, USA). Results are expressed as means, frequencies and percentages. Chi square was used to determine the level of significance of groups of categorical variables with $\mathrm{P}$ values $<0.05$ considered significant. Analysis of variance (ANOVA) was used to compare means of variables between the three age groups (number of drugs per prescription and number of antimicrobials).

Ethical Considerations: Ethical approval was obtained from the Hospital Research Ethics Committee before the commencement of the study.

\section{RESULTS}

A total of 600 prescriptions were collected out of which only 526 were suitable for analysis. There were $293(55.7 \%)$ prescriptions collected from male patients and $233(44.3 \%$ from female patients. The mean weight of the children was $10.4 \pm 4.7 \mathrm{~kg}$, and the median age was 12 months (range of 0.5 - 60 months). The patients were further classified into various age groups with neonates, infants and toddlers accounting for $2.5 \%, 47.7 \%$ and $49.8 \%$ respectively. Acute respiratory tract infections $(281 / 53.7 \%)$ were the most common diagnoses among children who presented at the outpatient clinic during the period of the study followed by malaria $(254 / 48.6 \%)$ and acute diarrhoea $(47 / 9 \%)$. The detailed analysis of the diagnoses is as in Table 1.

The total number of prescribed drugs was 1369 with a mean of $2.6 \pm 1.1$ drugs per prescription. The majority of the prescribed drugs were for oral administration $(1220 / 90.2 \%)$ while only $5.9 \%$ were given through the intra-muscular route. Overall, $13.5 \%$ of all encounters had an injection prescribed. The distribution of the number of prescribed drugs is shown in Figure 1. Prescribing by generic name was done in $68.9 \pm 26.5 \%$ of all prescribed medications.
Table 1: List of diagnoses of the children

\begin{tabular}{lcc}
\hline Diagnosis & N & \% \\
\hline Acute respiratory infections & 281 & 53.7 \\
Malaria & 254 & 48.6 \\
Acute Diarrhoea & 47 & 9 \\
Skin infection & 15 & 2.9 \\
Neonatal sepsis & 10 & 1.9 \\
Conjunctivitis & 11 & 2.1 \\
Chronic suppurative otitis media & 9 & 1.7 \\
Allergic dermatitis/Urticaria & 7 & 1.3 \\
Bronchial asthma & 5 & 1 \\
Urinary tract infection & 4 & 0.8 \\
Dog Bite & 3 & 0.6 \\
Others & 11 & 2.1 \\
\hline
\end{tabular}

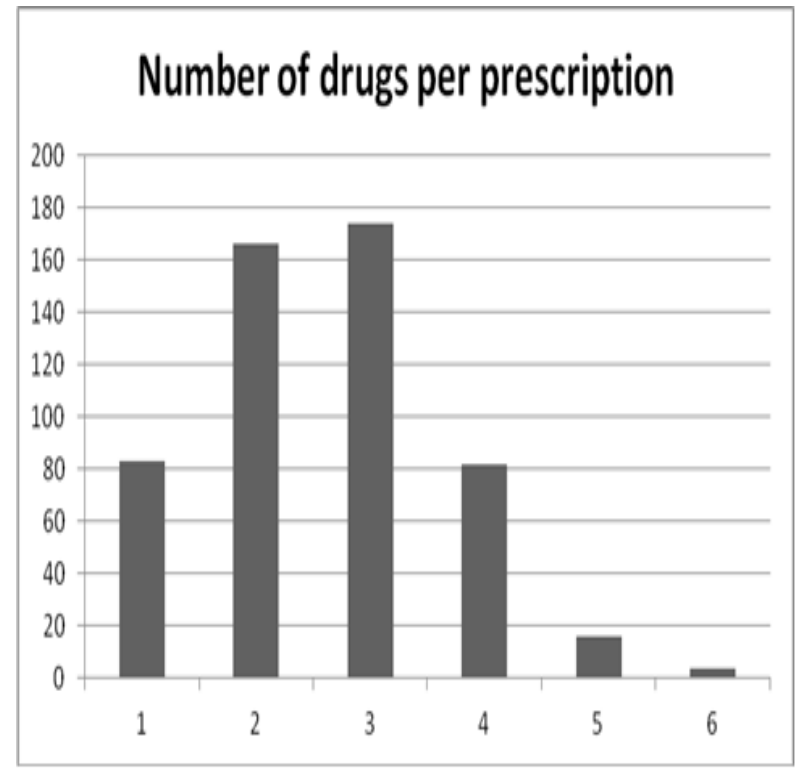

Figure 1: Frequency of prescribed drugs

A total of three hundred and seventy-four (71.1\%) patients had at least one antibiotic prescribed with antibiotics accounting for $28.2 \%$ of all drugs prescribed. Antibiotics from the penicillin group (71.2\%) were the most frequently prescribed followed by drugs from the cephalosporin $(15.8 \%)$ and macrolide groups $(8 \%)$. The majority of the patients with antibiotic prescribed had only one drug $(97.1 \%)$ while the remaining had two $(2.7 \%)$ and three $(0.2 \%)$ drugs respectively. The most frequently prescribed antibiotics were Amoxicillin (52.4\%), Amoxicillin/Clavulanic acid (19\%), Cefuroxime (15.2\%) and Erythromycin (7.2\%). The detailed pattern of antibiotic prescription is 
shown in Figure 2. One hundred and eighty-one patients $(34.4 \%)$ had co-prescription of antibiotics and anti-malarial drugs.

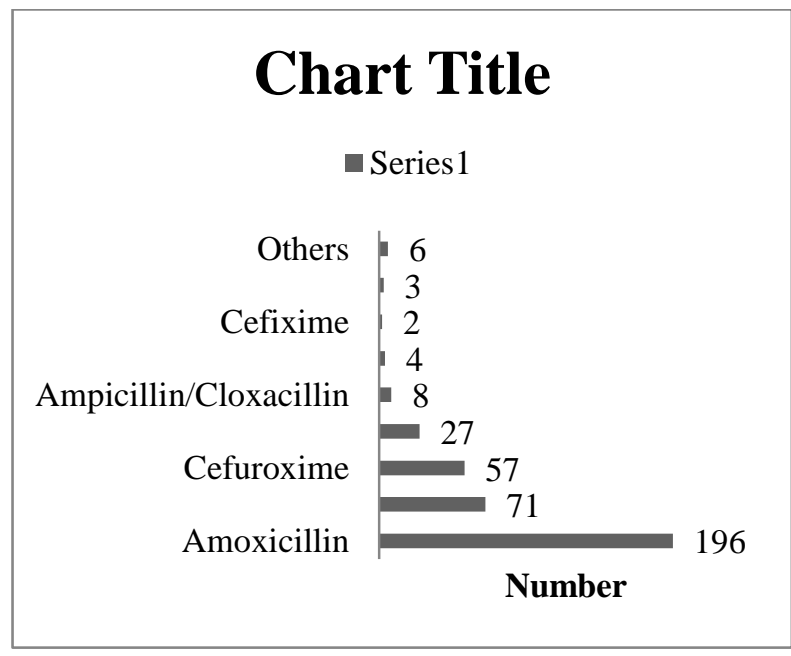

Figure 2: Prescription pattern of antibiotics

Three hundred and twelve children $(59.3 \%)$ had at least one anti-malarial drug prescribed. A total of 434 anti-malarial drugs were prescribed constituting $31.7 \%$ of all prescribed drugs. The fixed-drug combination Artemeter-Lumafantrine (AL) was the most prescribed in ninety-seven patients $(31.1 \%)$ followed by Dihydroartemisin + Piperaquine fixed-dose combination (92/29.5\%), Artesunate-Amodiaquine $(73 / 23.4 \%)$, ArtemeterAmodiaquine (29/9.3\%), Artemeter alone $(19 / 6.1 \%)$ (29/9.3\%), Artemeter alone Artermeter/Sulphadoxine/Pyrimethamine (1/0.3\%) and Amodiaquine alone (1/0.3\%). Analgesics drugs accounted for $6.1 \%$ of all prescriptions with Acetaminophen and Ibuprofen written in $90.4 \%$ and $9.6 \%$ respectively.

Of the 47 children with gastro-enteritis, 25 $(53.1 \%)$ had the probiotic Fluranom®, $36(76.6 \%)$ had Zinc supplementation while $21(44.7 \%)$ had Vitamin A supplementation. Oral rehydration salts were prescribed to $60(4.4 \%)$ children, Fluranom ${ }^{\circledR}$ consists of the anti-diarrhoeal micro-organism Saccharomyces boulardii used for the treatment of diarrhea of various aetiologies. Multi-vitamins and supplements accounted for $15.6 \%$ of all prescriptions with Ascorbic acid $(56.1 \%)$ being the most frequently prescribed drug in that category. About $60.4 \%$ of all prescribed medications were from the latest version of the Nigerian Essential Drug List with antibiotics and anti-malarials having $66.7 \%$ and $85.7 \%$ prescribed respectively.

A comparison of the means of prescribed medications among the three age groups of children using ANOVA showed statistical significance $(\mathrm{P}=0.003)$. Similar comparison using the mean prescribed antibiotics was not statistically significant $(\mathrm{P}=0.46)$.

\section{DISCUSSION}

There was a preponderance of male patients $(55.7 \%)$ in this study; this may be a reflection of the general sex distribution of the population as shown in the 2006 national population census where male children $(<14 \mathrm{yrs})$ represented $51.9 \%$ of that age group. It is also in keeping with findings from another Nigerian study among children below five years where males were $57.8 \%$ of the total number of participants (7). The proportion of children less than 1 year in our study was $50.2 \%$, close to $47.8 \%$ found in a study from the South-Eastern part of Nigeria (8).

The mean number of drugs per prescription found in our study was $2.6 \pm 1.1$. Risk et al, in a study among children in Gambia, found a mean of 2.2 while results from two similar studies in India showed a mean of 2.5 and 2.9 (11). Drugs as injections were prescribed in $5.9 \%$, slightly higher than $3.5 \%$ and $1.6 \%$ reported in other studies (12, 14). Prescribing by generic name is one of the major points of rational prescribing as it promotes improved access and affordability. In our study, $68.9 \%$ of all medications were written in their generic name, higher than $13.3 \%$ and $49.3 \%$ found in a study among Nigerian and Sudanese children respectively $(9,14)$. The level of generic prescribing in our study was however lower than the $87 \%$ found in a study carried out in public primary health care facilities in Tanzania (15). The differences in level of generic prescribing could be due to differential levels of training and experience of prescribers, influence of pharmaceutical detailing, availability and compliance with treatment guidelines.

The majority $(71.1 \%)$ of the patients in this study had at least an antibiotic prescribed with $28.2 \%$ of all prescribed drugs belonging to the antibiotic category. This very high level of 
antibiotic prescription among children is also reflected in studies from other developing countries as shown by the $81.3 \%, 79 \%, 63.4 \%$ and $51.6 \%$ found in studies conducted in Sudan, India, Gambia and Bahrain $(11,12,14,16)$. The reasons for this prescribing habit among prescribers may not be far-fetched; studies have shown that while the cause of most acute respiratory tract infections and diarrheal disease is indeed viral, many medical doctors and care-givers still treat with antibiotics $(17,18)$. Antibiotics from the penicillin group (Amoxicillin, Amoxicillin/Clavulanic acid) were the most commonly prescribed in this study followed by drugs from the cephalosporin class (Cefuroxime) and the macrolide Erythromycin. In a study by Sadoh et al conducted in the Southsouth region of Nigeria, Amoxicillin/Clavulanic acid was the most prescribed antibiotic for the treatment of upper respiratory tract infection (17). European studies on antibiotic use among children revealed a slightly different picture; Penicillin V was the most prescribed drug followed by macrolides in a Norwegian study while Phenoxymethylpenicillin was the most preferred in Denmark $(19,20)$. The use of the older generation of penicillins in parts of Europe is likely due to the strict restriction of access to antibiotics and compliance to treatment guidelines especially regarding treatment of acute (viral) respiratory tract infections. This generation of antibiotics is seen as ineffective again in Nigeria because of uncontrolled access to them and noncompliance to or even total lack of treatment guidelines for these common childhood conditions. Many times, care-givers have administered antibiotics before bringing the children to the hospital; this may explain the choice of more potent antibiotics by the prescribers in our study.

Over $99 \%$ of all anti-malarial prescriptions in our study contained an artemisinin derivative in combination with other drugs. This is a great improvement from the transitional years of antimalrial treatment policy change when the uptake of ACT was only $18.2 \%$ (21). The fixed-dose combination Artemeter-Lumefantrine (31.1\%) was the most commonly prescribed antimalarial in our study. This is much lower than $97.3 \%$ prescription rate for Artemeter-Lumefantrine reported in a recent study in Uganda (22). Availability of various types of Artemisinin-Based Combination
Therapy (ACT) in Nigeria without a strict preference for any of the combinations may account for the lower rate of AL prescription in our study. The prescription of artemisinin derivates as monotherapy $(6.1 \%)$ was very low in our study in contrast to the $14.3 \%, 24 \%$ and $33 \%$ found in studies from India and Ghana (23-25). The consequences of using the artemisinin derivates as monotherapy is that of development of resistance, worsening morbidity and mortality of children and increased healthcare costs from hospitalizations and loss of income by care-givers. The use of probiotics is a recent development in the prevention and treatment of diarrhea in children, and its effectiveness has been shown in some studies $(26,27)$. More than half of patients who presented with diarrhoeal disease had the probiotic Fluranorm ${ }^{\circledR}$ prescribed, in keeping with the findings of an Indian study on the management of diarrhoea (28). In our study, the majority of children with diarrhoeal disease had supplementation with Zinc, a practice that has been shown to reduce the overall incidence of the disease (29).

This study showed that over-prescription of antibiotics is a major issue among doctors in the paediatric outpatient clinic. There is a need to update prescribers on rational use of medicines, especially antibiotics to avoid development of resistance to these drugs. While the treatment of malaria in most of the patients followed the existing national malaria treatment guidelines, monotherapy of malaria with artemisinin derivatives need to be discouraged. The major drawback of the study is the fact that it was limited to only one centre; hence, the result cannot be generalized. However, the relatively large number of prescriptions and prescribers trained in different medical schools is a major strength of the study.

\section{ACKNOWLEDGEMENT}

The authors are grateful to the staff of the medical records unit, medical officers and residents of the paediatric outpatient clinic of the teaching hospital who assisted in the prescription selection and filling of the data collection forms.

\section{REFERENCES}

1. Federal Republic of Nigeria. 2006 Population and Housing Census. Abuja, Nigeria2010. 
2. Sule SS. Childhood morbidity and treatment pattern at the multipurpose health centre, Ilesa, Nigeria. Niger $J$ Med. 2003;12(3):145-9.

3. Aronson JK. Medication errors: what they are, how they happen, and how to avoid them. QJM. 2009;102(8):513-21.

4. Oshikoya KA, Ojo OI. Medication errors in paediatric outpatient prescriptions of a teaching hospital in Nigeria. Nig Q J Hosp Med. 2007;17(2):74-8.

5. Hersh AL, Jackson MA, Hicks LA. Principles of judicious antibiotic prescribing for upper respiratory tract infections in pediatrics. Pediatrics. 2013;132(6):1146-54.

6. World Health Organization. How to investigate drug use in Health Facilities: selected drug use indicators. Geneva1993 Contract No.: WHO/DAP/93.1.

7. Iloh GU, Amadi AN, Nwankwo BO, Ugwu VC. Common under-five morbidity in South-Eastern Nigeria: a study of its pattern in a rural mission general hospital in Imo State. Niger J Med. 2011 JanMar;20(1):99-104.

8. Nwolisa CE, Erinaugha AU, Ofoleta SI. Pattern of morbidity among pre-school children attending the children's outpatient clinic of Federal Medical Centre Owerri, Nigeria. Niger J Med. 2005;14(4):378-80.

9. Nwolisa CE, Erinaugha EU, Ofoleta SI. Prescribing practices of doctors attending to under fives in a children's outpatient clinic in Owerri, Nigeria. J Trop Pediatr. 2006;52(3):197-200.

10. Koura KG, Garcia A, Todoegnon B, Deloron P, Cot M, Faucher JF. Prevalence and factors related to antibiotic prescription in Benin: a school-based study. Acta Trop. 2013;127(2):87-90.

11. Risk R, Naismith H, Burnett A, Moore SE, Cham M, Unger S. Rational prescribing in paediatrics in a resource-limited setting. Arch Dis Child. 2013;98(7):503-9.

12. Pandey AA, Thakre SB, Bhatkule PR. Prescription analysis of pediatric outpatient practice in nagpur city. Indian J Community Med. 2010;35(1):70-3.

13. Karande S, Sankhe P, Kulkarni M. Patterns of prescription and drug dispensing. Indian $J$ Pediatr. $2005 ; 72(2): 117-21$.

14. Ahmed AM, Awad AI. Drug use practices at pediatric hospitals of Khartoum State, Sudan. Ann Pharmacother. 2010 ;44(12):1986-93.

15. Nsimba SE. Assessing prescribing and patient care indicators for children under five years old with malaria and other disease conditions in public primary health care facilities. Southeast Asian J Trop Med Public Health. 2006 ;37(1):206-14.

16. Senok AC, Ismaeel AY, Al-Qashar FA, Agab WA. Pattern of upper respiratory tract infections and physicians' antibiotic prescribing practices in Bahrain. Med Princ Pract. 2009;18(3):170-4.

17. Sadoh WE, Akinsete AM. Physicians management of sore throat in children in Benin City, Nigeria. Niger $J$ Clin Pract. 2009 ;12(4):407-11.
18. Ekwochi U, Chinawa JM, Obi I, Obu HA, Agwu S. Use and/or misuse of antibiotics in management of diarrhea among children in Enugu, Southeast Nigeria. J Trop Pediatr. 2013;59(4):314-6.

19. Fossum GH, Lindbaek M, Gjelstad S, Dalen I, Kvaerner KJ. Are children carrying the burden of broad-spectrum antibiotics in general practice? Prescription pattern for paediatric outpatients with respiratory tract infections in Norway. BMJ Open. 2013;3(1).

20. Lusini G, Lapi F, Sara B, Vannacci A, Mugelli A, Kragstrup J, et al. Antibiotic prescribing in paediatric populations: a comparison between Viareggio, Italy and Funen, Denmark. Eur J Public Health. 2009;19(4):434-8.

21. Etuk EU, Egua MA, Muhammad AA. Prescription pattern of antimalarial drugs in children below 5 years in a tertiary health institution in Nigeria. Ann Afr Med. 2008;7(1):24-8.

22. Sears D, Kigozi R, Mpimbaza A, Kakeeto S, Sserwanga A, Staedke SG, et al. Anti-malarial prescription practices among outpatients with laboratory-confirmed malaria in the setting of a health facility-based sentinel site surveillance system in Uganda. Malar J. 2013;12:252.

23. Mishra N, Anvikar AR, Shah NK, Kamal VK, Sharma SK, Srivastava HC, et al. Prescription practices and availability of artemisinin monotherapy in India: where do we stand? Malar J. 2011;10:360.

24. Dodoo AN, Fogg C, Asiimwe A, Nartey ET, Kodua A, Tenkorang $\mathrm{O}$, et al. Pattern of drug utilization for treatment of uncomplicated malaria in urban Ghana following national treatment policy change to artemisinin-combination therapy. Malar J. 2009;8:2.

25. Abdelgader TM, Ibrahim AM, Elmardi KA, Githinji S, Zurovac D, Snow RW, et al. Progress towards implementation of ACT malaria case-management in public health facilities in the Republic of Sudan: a cluster-sample survey. BMC Public Health. 2012;12:11.

26. Guandalini S. Probiotics for prevention and treatment of diarrhea. J Clin Gastroenterol. 2011;45 Suppl:S149.

27. Szajewska H, Mrukowicz JZ. Probiotics in the treatment and prevention of acute infectious diarrhea in infants and children: a systematic review of published randomized, double-blind, placebo-controlled trials. $J$ Pediatr Gastroenterol Nutr. 2001;33 Suppl 2:S17-25.

28. Chakraborti S, Barik KL, Singh AK, Nag SS. Prescribing practices of doctors in management of acute diarrhea. Indian Pediatr. 2011 ;48(10):811-2.

29. Yakoob MY, Theodoratou E, Jabeen A, Imdad A, Eisele TP, Ferguson J, et al. Preventive zinc supplementation in developing countries: impact on mortality and morbidity due to diarrhea, pneumonia and malaria. BMC Public Health. 2011;11(Suppl 3):S23. 